ORIGINAL ARTICLE

\title{
Early adjuvant use of nonopioids associated with reduced odds of serious postoperative opioid adverse events and need for rescue in children
}

\author{
Terri Voepel-Lewis ${ }^{1}$, Deborah Wagner ${ }^{1}$, Constance Burke ${ }^{1}$, Alan R. Tait ${ }^{1}$, Jennifer Hemberg ${ }^{2}$, \\ Elsa Pechlivanidis ${ }^{3}$, Shobha Malviya ${ }^{1} \&$ AkkeNeel Talsma $^{3}$ \\ 1 Department of Anesthesiology, University of Michigan Health Systems Ann Arbor, MI, USA \\ 2 Medical School, University of Michigan Health Systems Ann Arbor, MI, USA \\ 3 School of Nursing, University of Michigan Health Systems Ann Arbor, MI, USA
}

Keywords

acute pain; opioids; adverse events

\section{Correspondence \\ Terri Voepel-Lewis, MSN, RN \\ Department of Anesthesiology \\ University of Michgigan Health Systems \\ F4-917 Mott Hospital1540 E. Hospital Drive \\ Ann Arbor, MI 48109-4245, USA \\ Email: terriv@umich.edu}

Section Editor: Per-Arne Lonnqvist

Accepted 13 August 2012

doi:10.1111/pan.12026

\section{Summary}

Objectives: Examine factors associated with opioid adverse drug events (ADE) in children.

Specific Aims: Examine whether adjuvant nonopioid use is associated with a decreased probability of opioid-ADEs and need for rescue.

Background: Opioid-ADEs contribute to serious preventable harm for hospitalized children. Adjuvant nonopioid use may mitigate opioid risk postoperatively, yet few studies support this notion.

Method: This nested case-control study included children who required intervention or rescue from opioid-ADEs and procedure-matched controls. Data were recorded from medical records and primary outcomes included serious opioid-ADEs (over-sedation and respiratory depression) and need for rescue (e.g., naloxone, rapid response team). Hierarchical logistic regression (HLR) models examined relationships between factors and opioid-ADEs. Early clinical signs and symptoms of deterioration were examined.

Results: Twenty five children with opioid-ADEs and 98 children without events were included. ASA-PS remained an independent risk factor (odds ratio, $2.56[1.09,6.03] ; P=0.031$ ), while adjuvant nonopioids a risk reduction factor for opioid-ADEs (OR, 0.16 [0.05, 0.47]; $P=0.001$ ) and need for rescue $(0.14$ [0.04, 0.47]; $P=0.001)$. Supplemental oxygen use at PACU discharge was associated with an increased odds of opioid-ADEs (OR, $3.72[1.35$, 10.23]; $P=0.007)$ and need for rescue $(5.5[1.7,17.82] ; P=0.002)$.

Conclusions: Findings from this study suggest that strategies such as early use of adjuvant nonopioids may reduce risk of opioid-ADEs postoperatively. Furthermore, children who require supplemental oxygen early postoperatively may be at heightened risk of later events.

\section{Introduction}

Opioids are a primary source of adverse drug events (ADE) (1-3) contributing to serious preventable harm for hospitalized children (4). These agents have been associated with $20-51 \%$ of drug events and errors $(2,3)$. Associated morbidity, mortality, and resource utiliza- tion suggest an urgent need to decrease the occurrence of opioid-ADEs $(1,2,5)$.

The risk of opioid-ADEs may be higher early postoperatively and among children, given their variable response to medications (5). These risks were underscored by findings that $24 \%$ of children experienced a potentially serious postoperative opioid-ADE warranting intervention, rescue, 
or escalation of care (6). Such reports motivated calls for risk reduction strategies that include, enhanced risk stratification, monitoring, and assessment to ensure early detection and intervention (7-9) and consideration of opioidsparing techniques, such as adjuvant use of nonopioids (8). The insufficiency of risk stratification, to date, has led to an emphasis on comprehensive surveillance for early detection of deterioration $(9,10)$. Yet, ongoing adverse events and mortality in settings with continuous physiologic monitoring (11) suggest an influence of other factors, such as inattention or inadequate early intervention. It is therefore suggested that more emphasis be placed on strategies such as nonopioid use that may reduce opioid consumption (12); however, little is known about the impact of such strategies on safety. Such data are needed to bolster strategies that promote safety in vulnerable groups.

This nested case-control study examined the relationship between patient factors, treatment factors, and opioid outcomes in hospitalized children during the early postoperative period. It was hypothesized that early adjuvant use of nonopioids would reduce the risk of serious opioid-ADEs and need for rescue postoperatively. A secondary aim was to identify early clinical warning signs for opioid-ADEs and need for rescue.

\section{Materials and methods}

A partial analysis of data from an ongoing study of adverse postoperative events ${ }^{1}$ identified using rapid response team (RRT) records was conducted. With IRB approval and waiver of consent, the RRT, cardiopulmonary arrest, and pharmacy naloxone patient lists were reviewed to identify children who experienced rescue events on general care units within 1 week postoperatively. Controls were selected from the fixed base population of children receiving anesthetics over the same study period (January 2009-March 2011). Two controls were procedure-matched to every rescue case with the first selected at random from the procedure subgroup and the second using sequential numbering. Excluded were patients who were aged $>20$ years, had an outpatient or cardiothoracic procedure, underlying hematology/oncology condition or receiving opioids within 1 week preoperatively (i.e., potentially opioid tolerant), a nonopioid-related rescue event or an event in the intensive care unit (ICU), or never received opioids.

\footnotetext{
${ }^{1}$ Aims of larger study include describing the nature and timing of postoperative adverse events (PAE) in children; identify potential warning signs in the PACU; describe nurse assessment and staffing patterns associated with occurrence of PAE. All patients with RRT or CPA events are included in the larger study. This paper is the first manuscript from this study.
}

Trained assistants reviewed all records (i.e., automaticcapture electronic perioperative records, the electronic progress notes, medication order and administration data, and nursing flow-sheets) and recorded the following data: demographics and medical history, American Society of Anesthesiologists Physical Status (ASA-PS), procedure data, recovery progress over the first $48 \mathrm{~h}$ (vital signs [VS], oxygen saturation $\left[\mathrm{SpO}_{2}\right]$, fraction of inspired oxygen $\left[\mathrm{FiO}_{2}\right]$, pain $[0-10$ self-report numeric rating or FLACC behavioral scale] and sedation scores [University of Michigan Sedation Scale-UMSS, 0-4, where $4=$ unarousable] (13)), analgesics, and event details. To improve the reliable identification of opioid-ADEs, a structured trigger tool was modified from the Pediatric Trigger Toolkit Guide to Identifying ADEs in Children's Hospital Settings $(2,5)$ to include descriptors of interventions [e.g., naloxone, supplemental oxygen $\left(\mathrm{O}_{2}\right)$ ] and events (e.g., oxygen desaturation) potentially indicative of opioid- or sedative-related ADEs. Oxygen desaturation was defined as $\mathrm{SpO}_{2}<92 \%$, and oversedation as UMSS $\geq 2$ (i.e., moderate to deep sedation) accompanied by a progress note identifying excessive sleepiness warranting an intervention. Respiratory depression was defined as respiratory rate $\leq 16$ for infants $<6$ months of age, $\leq 12$ for $6 \mathrm{~m}-2$ years, $\leq 10$ for $3-10$ years, and $\leq 8$ for $>10$ years. Type and amount of analgesics (opioid and nonopioid) administered were recorded from the electronic medication administration record for every 8-h period on postoperative day 1 and for the 24-h period on day 2. Nonopioids included acetaminophen, ibuprofen, and ketorolac. For children with potential opioid-ADEs as identified from the trigger tool, analgesics and VS were recorded for $2 \mathrm{~h}$ increments preceding the event up to $12 \mathrm{~h}$ to establish temporal relationships to the event. Length of stay and mortality were noted.

\section{Definition of outcomes}

A secondary review was conducted by the primary investigator for all subjects to ensure completeness and to code opioid-ADEs based on a temporal relationship to opioids and sedatives. The primary outcome was Opioid-ADE that included children with potentially life-threatening events. The secondary outcome was need for rescue which included the subset of opioid-ADEs requiring naloxone, airway management, or transfer to an ICU. Children with these outcomes were compared with controls (children with no events), and those with need for rescue were further compared with children with threshold events (i.e., the subset of those needing an intermediate intervention such as $\mathrm{O}_{2}$, decrease or change in analgesic, or increased assessment). To ensure reliability of coding, event and medication data for all potential opioid-ADEs 
and a random selection of nonopioid-ADE cases were independently reviewed by two co-authors who coded the nature of each event (threshold vs. rescue vs. nonevent). Exact agreement occurred for all but two cases: one where a 2-year-old had severe desaturation after low doses of morphine and a benzodiazepine within $3 \mathrm{~h}$ of postanesthesia care unit discharge (PACU DC), and the other an infant with sleep apnea who had oxygen desaturation $4 \mathrm{~h}$ after a low dose of an oral opioid, responding to naloxone. These cases were considered potential opioid-ADEs and included in the case group.

\section{Statistical analysis}

All data were analyzed using sPss statistical software (v.18, Chicago, IL, USA). Morphine equivalents $\left(\mathrm{mg} \cdot \mathrm{kg}^{-1}\right)$ were calculated using standard equianalgesic conversions for intraoperative, PACU, and post-PACU doses administered, and for initial opioid doses ordered. Surgical severity was scored using a validated tool where $\mathrm{A}=$ noninvasive (e.g., superficial peripheral), B = invasive with moderate blood loss or airway procedure (e.g., incision and drainage, tonsillectomy), and $\mathrm{C}=$ significantly invasive (e.g., spine fusion) (14) and inter-rater reliability was excellent $(\alpha=0.915)$. Data are described as; $n(\%)$; means \pm SD or $95 \%$ confidence intervals $[\mathrm{CI}]$, and odds ratios [CI]. Chi square with Fisher's exact tests compared binominal data and unpaired $t$-tests with Levene's tests compared continuous data.

Hierarchical logistic regression (HLR) models with forced entry of independent variables examined predictors for opioid-ADE and need for rescue. Patient factors that were different between groups $(P<0.10$; two-sided $)$ were entered at the first blocks, followed by the nonopioid factor at the final block to determine the relative contribution of nonopioids to the outcomes. The models were subsequently evaluated for the ability to discriminate opioid-ADEs or need for rescue using receiver operating characteristic curves, with the predicted probabilities as the test variable (15).

Extreme clinical signs (e.g., low $\mathrm{SpO}_{2}$ ) during PACU, at PACU DC and during the first $8 \mathrm{~h}$ as well as opioid consumption over time, were compared between groups using Kruskal-Wallis one-way analysis of variance with Friedman's tests, because assumptions of normality were not met (Kolmogorov-Smirnov statistics $<0.05$ ). $P$ values $<0.05$ (two-sided) were considered significant.

\section{Sample size}

As the derived sample was based on a fixed data set, post hoc analysis revealed that a sample of 26 children per group would have $80 \%$ power $(P=0.05)$ to detect what Cohen describes as a large effect size $(0.5)$ between children with opioid-ADEs and those without these events (16).

\section{Results}

Figure 1 depicts the selection of cases. Sixteen of 86 patients with rescue events met inclusion criteria and had experienced an opioid-ADE. The trigger tool identified nine additional children with threshold events from the case-matched selection. The resultant study group included the 25 children with opioid-ADEs, and the control group comprised 98 who received opioids without event.

\section{Event description}

Seventeen of the opioid-ADEs $(68 \%)$ involved oxygen desaturation, $13(52 \%)$ over-sedation, $7(28 \%)$ respiratory depression, $5(20 \%)$ agitation, and $1(4 \%)$ hypotension. Fourteen cases $(56 \%)$ were treated with supplemental $\mathrm{O}_{2}$,

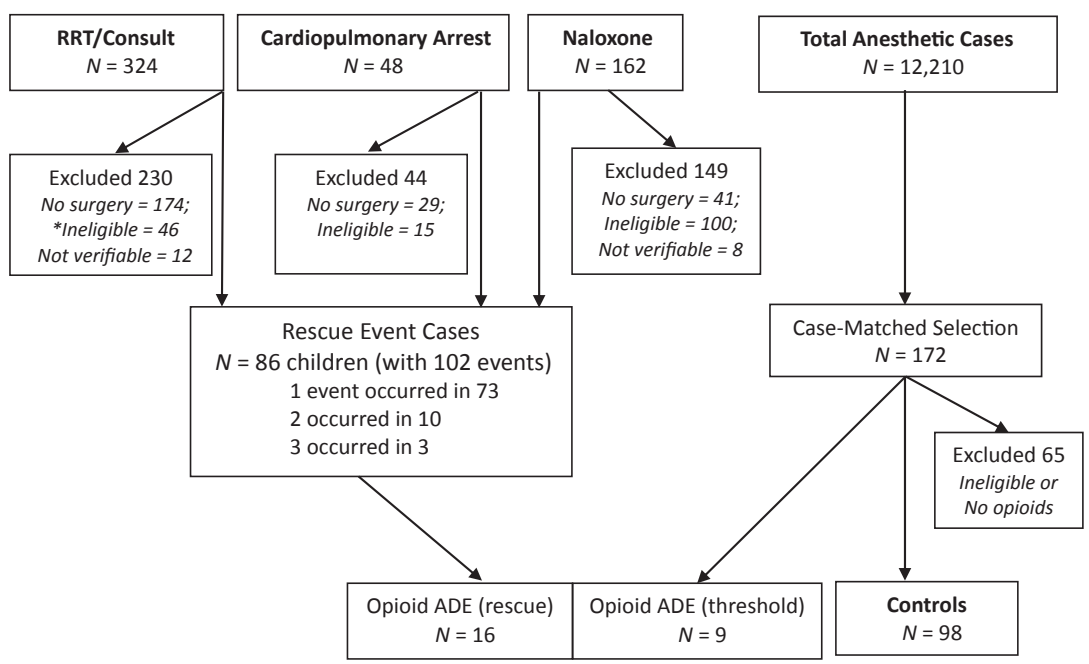

Figure 1 Selection of study subjects. ${ }^{\text {a }}$ Ineligible = cardiac surgery, event occurred preoperatively, event because of nonclinical reason (e.g., blood draw assistance), patient $>20$ years of age, hematologic/oncologic condition, no opioids received. ${ }^{\mathrm{b}}$ Population of noncardiac, admissions after surgery only, $\leq 20$ years of age. ${ }^{\text {CAdverse }}$ drug event $=$ Serious event associated or potentially associated with drug administration. 
$13(52 \%)$ naloxone, and $13(52 \%)$ a decrease in opioid dose (the difference in preevent opioid use, however, was not significantly different compared with $0-8,8-16$, or 16-24 h use; $P=0.238$ ). Less common interventions included $7(28 \%)$ medication changes, $5(20 \%)$ airway management (i.e., three cases jaw thrust, one nasopharyngeal airway, one endotracheal intubation), and $5(20 \%)$ required escalation of care to a moderate care or ICU setting. The time from PACU DC to an opioid-ADE was $17.85 \mathrm{~h}[4.5,31.2]$, and the time to threshold vs rescue events was not different $(13.9[5.03,22.79]$ vs. 13.06 [1.00, $25.16]$ h, respectively, $P=0.65$ ). The opioid-ADE group had more nursing assessments per shift compared with controls $(4.1[3.2,5.0]$ vs. $3.3[2.8,3.8] ; P<0.001)$, but a similar use of continuous pulse oximetry monitoring $(88 \%$ vs. $72 \%, P=0.124)$. The hospital length of stay was longer for children with opioid-ADE $(8.7[5.24,12.12]$ vs. controls $5.40[2.36,8.44] ; P<0.001)$, and there were no deaths or permanent injury.

\section{Aim 1: Association between nonopioids and opioid-ADEs and need for rescue}

Univariate comparisons found differences in ASA-PS, musculoskeletal condition, use of IV-NCA, and continuous opioid infusion, but not in perioperative or postoperative opioid use during the first $48 \mathrm{~h}$ (Table 1). Overall morphine equivalents during this timeframe were not significantly different between children who received adjuvant nonopioids and those who did not $(0.005$ [0.004, $0.007]$ vs. 0.008 [0.001, 0.012] $\mathrm{mg} \cdot \mathrm{kg}^{-1} \cdot \mathrm{h}^{-1} ; P=0.408$ ); however, the opioid-ADE group had lower odds of having received adjuvant nonopioids. The HLR model coefficients confirmed the goodness of fit and supported the hypothesis that early nonopioid use was associated with a decreased probability of opioid-ADEs, and the c-statistic supported the model's prognostic ability (Table 2).

Compared with controls, the subgroup of children who needed rescue was less likely to have had an ASAPS of $1(0.78$ [0.70, 0.86]; $P=0.039]$ and less likely to have received adjuvant nonopioids (OR 0.21 [0.07, 0.63 ], $P=0.003$ ). Use of continuous infusion was $38 \%$ for the rescue group compared with $19 \%$ for controls $(P=0.104)$. All other patient and treatment factors were not significantly different between the rescues and controls (Appendix S1). An HLR analysis included ASA and continuous infusion as independent variables in the first block and adjuvant nonopioids in the second. The addition of the nonopioid factor significantly improved the model fit $\left(r^{2}\right.$ change $\left.=0.158\right)$ and was associated with an $86 \%$ reduced odds of need for rescue (Table 3). The prognostic ability of this model to detect the outcome was supported by the c-statistic.

\section{Aim 2: Early clinical predictors of opioid-ADEs and need for rescue}

There were no differences between groups in documented HR or RR during the PACU stay or in the first $8 \mathrm{~h}$ postoperatively $(P>0.169)$ (Appendix S2). Table 4 presents a description of pain and UMSS scores, $\mathrm{SpO}_{2}$ and supplemental $\mathrm{O}_{2}$ requirements between children with and without opioid-ADEs. The opioid-ADE group was nearly four times as likely to have required $\mathrm{O}_{2}$ at PACU DC, and more than 10 times as likely to have needed it preevent.

Children in the rescue group had a significantly lower $\mathrm{SpO}_{2}$ in the $12 \mathrm{~h}$ prior to the event compared with the threshold group ( $86.56 \pm 8.2$ vs. $93.67 \pm 3.91 ; P=0.008)$, and compared with all others, were more likely to have needed $\mathrm{O}_{2}$ at PACU DC (OR $5.5[1.7,17.82] ; P=0.002$ ) and preevent $(27.86[7.04,110.28] P<0.001)$. The need for $\mathrm{O}_{2}$ was not significantly different between threshold and rescue groups $(22 \%$ vs. $50 \%, P=0.228$ at $\mathrm{PACU}$ DC and $44 \%$ vs. $81 \%, P=0.087$ preevent).

\section{Discussion}

Findings supported the hypotheses that early use of adjuvant nonopioids was associated with a lower probability of opioid-ADE and need for rescue in this setting. Furthermore, lower $\mathrm{SpO}_{2}$, higher sedation scores, and use of supplemental $\mathrm{O}_{2}$ at PACU DC and in the early postoperative period were early signs of later deterioration, suggesting the need for vigilance and, perhaps, lowering opioid doses in the presence of these signs.

The mitigating effect of adjuvant nonopioids in this study is particularly noteworthy. Recent reviews and studies in adult settings suggest that addition of nonopioids reduces narcotic consumption, enhances analgesia, and lessens side effects such as nausea and vomiting (17-20). However, the attenuating effects of nonopioids for respiratory depression or sedation have not been well documented, with some studies suggesting no substantial reduction $(21,22)$ and others showing reduced sedation but not respiratory depression (23). Limited data have yielded similar findings for children, supporting the opioid-sparing and analgesic effects of nonopioids, but lacking support for serious event mitigation (24). Conflicting findings between reports may be due, in part, to variations in methods used to detect and define serious opioidADEs (23). Despite such variations, ASA practice guidelines state that 'whenever possible, anesthesiologists should employ multimodal pain management therapy' (25). Given the strong association of this factor with a lower odds of events and need for rescue, broader implementation may be warranted. No 
Table 1 Characteristics and description of treatment(s) between groups

\begin{tabular}{|c|c|c|c|c|}
\hline & Opioid-ADE cases $N=25$ & Controls $N=98$ & $P$ values & OR $[95 \% \mathrm{Cl}]$ \\
\hline Age (mean years \pm SD) & $8.08[5.4,10.7]$ & $6.23[5.0,7.5]$ & 0.184 & NA \\
\hline Weight (mean $\mathrm{kg} \pm \mathrm{SD}$ ) & $28.66[20.3,37.02]$ & $26.08[21.4,30.7]$ & 0.611 & NA \\
\hline Gender-male $n(\%)$ & $14(56)$ & $53(54)$ & 0.863 & $0.93[0.38,2.24]$ \\
\hline Caucasian race $n(\%)$ & $19(76)$ & $78(80)$ & 0.963 & $0.97[0.32,2.93]$ \\
\hline Hispanic $n(\%)$ & 0 & $4(4)$ & 1.00 & $0.96[0.92,1.00]$ \\
\hline ASA $1 n(\%)$ & 0 & $22(22)$ & 0.007 & $0.78[0.70,0.86]$ \\
\hline ASA $2 n(\%)$ & $12(48)$ & $43(44)$ & 0.711 & $1.18[0.49,2.85]$ \\
\hline ASA $3 n(\%)$ & $13(52)$ & $32(33)$ & 0.073 & $2.23[0.92,5.45]$ \\
\hline ASA $4 n(\%)$ & 0 & $1(1)$ & 1.000 & $0.97[0.97,1.01]$ \\
\hline Emergent case & $1(4 \%)$ & $9(9 \%)$ & 0.685 & $0.41[0.05,3.41]$ \\
\hline \multicolumn{5}{|l|}{ Comorbid conditions $n(\%)$} \\
\hline Respiratory & $9(36)$ & $22(22)$ & 0.164 & $1.94[0.76,4.98]$ \\
\hline Cardiovascular & $4(16)$ & $13(13)$ & 0.748 & $1.25[0.37,4.21]$ \\
\hline Obstructive sleep apnea & $6(24)$ & $15(15)$ & 0.302 & $1.75[0.60,5.09]$ \\
\hline Neurologic & $13(52)$ & 38 (39) & 0.231 & $1.71[0.71,4.14]$ \\
\hline Obesity & $2(8)$ & $5(5)$ & 0.629 & $1.62[0.30,8.87]$ \\
\hline Hepato-renal & $2(8)$ & $10(10)$ & 1.00 & $0.77[0.16,3.74]$ \\
\hline Musculoskeletal & $13(52)$ & $21(21)$ & 0.002 & $3.97[1.58,9.98]$ \\
\hline Genito-urinary & $3(12)$ & $3(3)$ & 0.098 & $4.32[0.82,22.85]$ \\
\hline Gastrointestinal & $8(32)$ & $26(27)$ & 0.585 & $1.30[0.50,3.38]$ \\
\hline \multicolumn{5}{|l|}{ Surgical severity code (14) $n(\%)$} \\
\hline A - Minimally invasive & $5(20)$ & $30(31)$ & 0.333 & $0.57[0.19,1.65]$ \\
\hline B - Moderately invasive & $11(44)$ & $47(48)$ & 0.723 & $0.85[0.35,2.06]$ \\
\hline C - Significantly invasive & $9(36)$ & $21(21)$ & 0.130 & $2.06[0.80,5.33]$ \\
\hline \multicolumn{5}{|l|}{ Perioperative opioids a mean $[95 \% \mathrm{Cl}]$} \\
\hline Intraoperative opioid $\mathrm{mg} \cdot \mathrm{kg}^{-1} \cdot \mathrm{h}^{-1}$ & $0.087[0.06,0.12]$ & $0.079[0.07,0.09]$ & 0.535 & NA \\
\hline PACU morphine $\mathrm{mg} \cdot \mathrm{kg}^{-1}$ & $0.098[-0.06,0.26]$ & $0.076[0.003,0.15]$ & 0.800 & NA \\
\hline \multicolumn{5}{|l|}{ Post-PACU IV morphine $\mathrm{mg} \cdot \mathrm{kg}^{-1} \mathrm{~b}$} \\
\hline 0-8 h postoperative & $0.095[0.04,0.15]$ & $0.072[0.05,0.09]$ & 0.253 & NA \\
\hline 8-16 h postoperative & $0.10[0.02,0.19]$ & $0.06[0.04,0.08]$ & 0.407 & NA \\
\hline 16-24 h postoperative & $0.09[-0.004,0.19]$ & $0.06[0.04,0.07]$ & 0.531 & NA \\
\hline 24-48 h postoperative & $0.26[-0.04,0.56]$ & $0.12[0.08,0.17]$ & 0.421 & NA \\
\hline \multicolumn{5}{|c|}{ Postoperative analgesic method $n(\%)$; ordered dose morphine equivalent $\cdot \mathrm{kg}^{-1}[\mathrm{Cl}]$} \\
\hline Oral opioid & $2(8) ; 0.02[0.00,0.07]$ & $20(20) ; 0.05[0.04,0.06]$ & $\begin{array}{c}0.241 \\
0.032\end{array}$ & $0.34[0.07,1.56]$ \\
\hline IV prn & $9(36) ; 0.06[0.04,0.08]$ & $25(26) ; 0.07[0.06,0.07]$ & $\begin{array}{c}0.295 \\
0.293\end{array}$ & $1.64[0.65,4.18]$ \\
\hline IV PCA & $4(16) ; 0.019[0.015,0.024]$ & $11(11) ; 0.016[0.015,0.018]$ & $\begin{array}{c}0.504 \\
0.291\end{array}$ & $1.51[0.44,5.20]$ \\
\hline IV NCA & $6(24) ; 0.02[0.015,0.025]$ & $8(8) ; 0.018[0.014,0.02]$ & $\begin{array}{c}0.026 \\
0.606\end{array}$ & $3.55[1.10,11.43]$ \\
\hline Epidural & $4(16) ; 0.21[0.17,0.24]$ & 7 (7); $0.20[0.14,0.26]$ & $\begin{array}{c}0.232 \\
0.705\end{array}$ & $2.48[0.66,9.24]$ \\
\hline Continuous opioid infusion $n(\%)$ & $11(44) ; 0.01[0.007,0.01]$ & 19 (19); $0.010[0.008,0.01]$ & $\begin{array}{c}0.011 \\
0.176\end{array}$ & $3.27[1.28,8.32]$ \\
\hline Benzodiazepines $n(\%)$ & $8(32)$ & $16(16)$ & 0.078 & $2.41[0.89,6.53]$ \\
\hline Adjuvant nonopioid $n(\%)$ & $11(44)$ & $77(79)$ & 0.001 & $0.21[0.09,0.54]$ \\
\hline
\end{tabular}

PACU, postanesthesia care unit; IV, intravenous; PCA, patient-controlled analgesia; NCA, nurse-controlled analgesia.

${ }^{a}$ Opioids represent morphine equivalents (from all routes) based on common equi-analgesic calculations.

${ }^{b}$ Morphine equivalents by $8 \mathrm{~h}$ increments, postoperative day 1 and overall on day 2 ; there were no significant changes over time between or within groups ( $P>0.10$ all comparisons).

attempt was made to stratify by type of nonopioid in this study, and most commonly acetaminophen (10$15 \mathrm{mg} \cdot \mathrm{kg}^{-1}$ ) was administered. A previous review of adult studies suggested no clear differences in opioid- or ADE-sparing effects between NSAIDs, paracetamol, and cyclo-oxygenase 2 inhibitors (17); however, the choice should be made in consideration of the risk/benefit profiles of these and other adjuvants (26). 
Table 2 Patient and treatment predictors of opioid-ADE

\begin{tabular}{|c|c|c|c|c|}
\hline & $B(S E)$ & Wald & Significance & OR $[95 \% \mathrm{Cl}]$ \\
\hline \multicolumn{5}{|c|}{ Block 1 Patient factors (Nagelkerke $r^{2}=0.151 ;$ Hosmer-Lemeshow $[\mathrm{HL}] 0.358 ; P=0.002$ ) } \\
\hline ASA-PS & $0.67(0.35)$ & 3.61 & 0.057 & $1.95[0.98,3.9]$ \\
\hline Musculoskeletal & $1.21(0.48)$ & 6.32 & 0.012 & $3.36[1.31,8.67]$ \\
\hline \multicolumn{5}{|c|}{ Block 2 Opioid factors $\left(r^{2}=0.198 ; \mathrm{HL}=0.619 ; P=0.246\right)$} \\
\hline ASA-PS & $0.84(0.40)$ & 4.53 & 0.033 & $2.32[1.07,5.04]$ \\
\hline Musculoskeletal & $0.58(0.58)$ & 1.00 & 0.316 & $1.79[0.57,5.60]$ \\
\hline IV-NCA & $0.43(0.71)$ & 0.38 & 0.540 & $1.54[0.39,6.15]$ \\
\hline Continuous infusion & $0.94(0.64)$ & 2.17 & 0.141 & $2.56[0.73,8.91]$ \\
\hline Benzodiazepine & $0.40(0.58)$ & 0.49 & 0.483 & $1.50[0.48,4.63]$ \\
\hline \multicolumn{5}{|c|}{ Block 3 Nonopioid Factor $\left(r^{2}=0.325 ; \mathrm{HL}=0.741 ; P=0.001\right)$} \\
\hline ASA-PS & $0.94(0.44)$ & 4.66 & 0.031 & $2.56[1.09,6.03]$ \\
\hline Musculoskeletal & $0.46(0.61)$ & 0.57 & 0.452 & $1.58[0.48,5.17]$ \\
\hline IV-NCA & $0.69(0.73)$ & 0.90 & 0.343 & $2.00[0.48,8.34]$ \\
\hline Continuous infusion & $0.95(0.68)$ & 1.97 & 0.160 & $2.58[0.69,9.70]$ \\
\hline Benzodiazepine & $0.91(0.62)$ & 2.15 & 0.143 & $2.48[0.74,8.36]$ \\
\hline Adjuvant nonopioid & $-1.83(0.55)$ & 10.98 & 0.001 & $0.16[0.05,0.47]$ \\
\hline
\end{tabular}

Posthoc c-statistic $=0.825$, standard error $=0.040[95 \% \mathrm{Cl}, 0.747,0.903] ; P<0.001$ (calculated using the predicted probabilities as the test variable in a receiver operating characteristic curve).

Table 3 Patient and treatment predictors of need for rescue

\begin{tabular}{|c|c|c|c|c|}
\hline & $\mathrm{B}(\mathrm{SE})$ & Wald & Significance & OR $[95 \% \mathrm{Cl}]$ \\
\hline \multicolumn{5}{|c|}{ Block 1 (Nagelkerke $r^{2}=0.093 ;$ Hosmer-Lemeshow [HL] 0.220; $P=0.049$ ) } \\
\hline ASA-PS & $0.754(0.41)$ & 3.36 & 0.067 & $2.13[0.95,4.76]$ \\
\hline Continuous infusion & $1.15(0.61)$ & 3.60 & 0.058 & $3.16[0.96,10.40]$ \\
\hline \multicolumn{5}{|c|}{ Block $2\left(r^{2}=0.251 ; \mathrm{HL}=0.43 ; P=0.001\right)$} \\
\hline ASA-PS & $0.89(0.46)$ & 3.78 & 0.052 & $2.44[0.99,5.99]$ \\
\hline Continuous infusion & $1.42(0.68)$ & 4.37 & 0.037 & $1.09[1.09,15.69]$ \\
\hline Adjuvant nonopioid & $-1.97(0.61)$ & 10.24 & 0.001 & $0.14[0.04,0.47]$ \\
\hline
\end{tabular}

Posthoc c-statistic $=0.82[0.735,0.905] ; P<0.001$ (calculated using the predicted probabilities as the test variable in a receiver operating characteristic curve).

A recent meta-analysis found insufficient dosing of NSAIDs in most studies in children, suggesting that better dosing practices may further improve opioidsparing and ADE reduction effects (24). Although nonopioid use was not associated with a lower dose of opioid in our study, the study was likely underpowered to detect an opioid-sparing effect.

Difficulties predicting which patients are at greatest risk of opioid-ADE emphasize the importance of careful surveillance and early detection of deterioration. While, not surprisingly, high sedation scores and lower $\mathrm{SpO}_{2}$ preceded threshold and rescue events, an important finding was the strength of the relationship between opioidADEs and supplemental $\mathrm{O}_{2}$ use both at PACU DC and early postoperatively. The trend toward lower $\mathrm{SpO}_{2}$ during the PACU stay may have been masked by the use of supplemental $\mathrm{O}_{2}$, because its use was higher but $\mathrm{SpO}_{2}$ similar by the time of DC. The ability of low-flow $\mathrm{O}_{2}$ to conceal respiratory depression (RD) is well documented (27), with evidence of delays from the earliest signs (i.e., bradypnea or depressed end-tidal carbon dioxide) to the onset of hypoxemia $(28,29)$ and impaired recognition in its presence (30). The wide range of time to recognition of events in this setting may reflect such delayed recognition. Of note, the frequency of nursing assessments was higher for children with opioid-ADEs suggesting an increased vigilance that may have prevented further deterioration in this setting. These findings suggest the need for a tiered approach to managing early signs of deterioration, starting with decreasing the opioid dose and enhanced vigilance, and a more restrictive or judicious use of supplemental $\mathrm{O}_{2}$. The APSF further suggests the use of capnography when supplemental $\mathrm{O}_{2}$ is in use (9).

The potential for selection bias in this study cannot be dismissed, despite attempts to minimize this by reviewing all records across samples and by randomly selecting controls from procedure sub-groups. The potential for misclassification of opioid-ADEs was reduced by using a trigger tool similar to those that improved the reliability of ADE identification in other studies (31) and by independent review by three investigators. Misclassification of comorbid conditions, such as OSA, was possible 
Table 4 Early clinical signs and symptoms between groups (data presented as mean [95\% confidence interval])

\begin{tabular}{|c|c|c|c|c|}
\hline & Preoperatively & PACU & PACU DC & $\mathrm{POD} 1^{\mathrm{a}}$ \\
\hline \multicolumn{5}{|l|}{ Low $\mathrm{SpO}_{2}$} \\
\hline Opioid-ADE & $97.7[96.6,98.9]$ & $91.6[87.2,95.9]$ & $96.0[94.1,97.8]$ & $88.6[85.4,91.8]$ \\
\hline Control & $97.7[96.3,99.2]$ & $95.0[93.9,96.2]$ & $97.5[97.1,97.9]$ & $95.7[95.2,96.2]$ \\
\hline$P$ value & 0.284 & 0.099 & 0.101 & $<0.001$ \\
\hline \multicolumn{5}{|l|}{ High UMSS ${ }^{b}$} \\
\hline Opioid-ADE & NA & $2.4[1.9,2.8]$ & $1.3[1.0,1.6]$ & $1.9[1.6,2.2]$ \\
\hline Control & NA & $2.5[2.3,2.7]$ & $1.1[0.9,1.3]$ & $1.3[1.1,1.5]$ \\
\hline$P$ value & & 0.486 & 0.267 & 0.002 \\
\hline \multicolumn{5}{|l|}{ Highest Pain Score ${ }^{c}$} \\
\hline Opioid-ADE & NA & $1.8[0.5,3.1]$ & $0.28[0,0.8]$ & $4.1[2.9,5.3]$ \\
\hline Control & NA & $2.9[2.3,3.6]$ & $0.79[0.4,1.2]$ & $3.7[3.2,4.3]$ \\
\hline$P$ value & & 0.117 & 0.130 & 0.678 \\
\hline \multicolumn{5}{|c|}{ Supplemental $\mathrm{O}_{2}$ use $n(\%) ; \mathrm{FiO}_{2}$} \\
\hline Opioid-ADE & RA & 19 (83); $0.44[0.31,0.57]$ & $9(39) ; 0.27[0.21,0.34]$ & $17(68) ; 0.35[0.26,0.47]$ \\
\hline Control & RA & 68 (72); 0.39 [0.33, 0.44] & 14 (15); $0.23[0.21,0.25]$ & $10(11) ; 0.22[0.21,0.23]$ \\
\hline Odds ratio $[95 \% \mathrm{Cl}] ; P$ value & & $1.89[0.59,6.06] ; 0.408$ & $3.72[1.35,10.23] ; 0.007$ & $10.82[3.88,30.19] ;<0.001$ \\
\hline
\end{tabular}

$\mathrm{PACU}$, post-anesthesia care unit; $\mathrm{DC}$, discharge; $\mathrm{POD}$, postoperative day; $\mathrm{ADE}$, adverse drug event; $\mathrm{O}_{2}$, oxygen, $\mathrm{FiO}_{2}$, fraction of inspired oxygen; $\mathrm{SpO}_{2}$, oxygen saturation.

a $V$ alues for Opioid-ADE group reflect only those prior to event.

${ }^{b}$ UMSS $=$ University of Michigan Sedation Scale $(0=$ awake, alert, $1=$ sleepy, but awake, 2 =arouses to voice or light tactile stimuli, $3=$ arouses to deep physical stimuli, $4=$ unarousable) (13).

${ }^{\mathrm{c}}$ Highest documented pain score (i.e., $0-10$, where $0=$ no pain, $10=$ worst pain).

because these were based on the documented history and physical exams. To minimize reporting error, comorbidities were coded from preoperative anesthesia and surgery records, as well as hospital admission notes. Given the small number of adverse events, it is possible that the model coefficients were overestimated. However, the goodness of fit and c-statistic showed excellent model performance. The finding that opioid consumption did not differ between groups must be cautiously interpreted. Morphine equivalents were calculated using standard equianalgesic conversions, yet may introduce confounding given differences in pharmacokinetics and active metabolites between agents. Furthermore, the retrospective nature of this study precluded the ability to compare dosage differences over time between groups given the variable time to events, dosage changes in response to events (event group) and over time (in control group). Prospective study controlling for agent and time is warranted to better examine the impact of opioid consumption. Lastly, the small samples of children with rescue and threshold events were likely underpowered to detect significant differences, and lastly, the likely contribution of other confounders that were overlooked cannot be dismissed. Further study in larger samples is warranted to further improve risk stratification.

In summary, findings suggest that early administration of adjuvant nonopioids may reduce the risk of serious opioid-ADEs in hospitalized children postoperatively. Enhanced vigilance and attention to early clinical signs of deterioration, including the child's need for supplemental $\mathrm{O}_{2}$, may facilitate early and effective intervention that prevents failure to rescue from opioid-ADEs. These findings lend support for recent calls to implement opioid risk mitigation strategies for hospitalized patients (32).

\section{Acknowledgment}

This work was funded in part by a Student Award from the Blue Cross Blue Shield of Michigan Foundation.

\section{Funding}

This reserach was carried out without funding.

\section{Conflict of interest}

No conflict of interest declared.

\section{Supporting information}

Additional Supporting Information may be found in the online version of this article:

Appendix S1 Threshold vs. rescue events.

Appendix S2 Vital sign extremes in the groups.

Please note: Wiley-Blackwell are not responsible for the content or functionality of any supporting materials supplied by the authors. Any queries (other than missing material) should be directed to the corresponding author for the article. 


\section{References}

1 Holdsworth MT, Fichtl RE, Behta M et al. Incidence and impact of adverse drug events in pediatric inpatients. Arch Pediatr Adolesc Med 2003; 157: 60-65.

2 Takata GS, Mason W, Taketomo C et al. Development, testing, and findings of a pediatric-focused trigger tool to identify medication-related harm in US children's hospitals. Pediatrics 2008; 121: e927-e935.

3 Kaushal R, Bates DW, Landrigan C et al. Medication errors and adverse drug events in pediatric inpatients. $J A M A 2001 ; \mathbf{2 8 5}$ : 2114-2120.

4 Morton NS, Errera A. APA national audit of pediatric opioid infusions. Pediatr Anesth 2010; 20: 119-125.

5 Pediatric Affinity Group. How-to-Guide Pediatric Supplement: Adverse Drug Events. Boston, MA: Institute for Healthcare Improvement (IHI), 2002.

6 Voepel-Lewis T, Marinkovic A, Kostrzewa A et al. The prevalence of and risk factors for adverse events in children receiving patient-controlled analgesia by proxy or patient-controlled analgesia after surgery. Anesth Analg 2008; 107: 70-75.

7 Institute of Safe Medication Practices. Safety Issues With Patient-Controlled Analgesia Part 1: How Errors Occur. Huntingdon Valley, PA: ISMP, July 10, 2003.

8 Jarzyna D, Jungquist CR, Pasero C et al. American Society for Pain Management Nursing guidelines on monitoring for opioid-induced sedation and respiratory depression. Pain Manag Nurs 2011; 12: 118-145.

9 Weinger M, Lee L. No patient shall be harmed by opioid-induced respiratory depression. APSF Newsletter: Official journal of the Anesthesia Patient Safety Foundation 2011; 26: 21-40.

10 Taenzer AH, Pyke JB, McGrath SP. A review of current and emerging approaches to address failure-to-rescue. Anesthesiology 2011; 115: 421-431.

11 Ochroch EA, Russell MW, Hanson WC et al. The impact of continuous pulse oximetry monitoring on intensive care unit admissions from a postsurgical care floor. Anesth Analg 2006; 102: 868-875.

12 Marwah R, Carvalho B. Reader stresses use of multimodal analgesia to decrease risk of opioid-induced respiratory depression. APSF Newsletter: Official journal of the Anesthesia Patient Safety Foundation 2012; 26: 62.

13 Malviya S, Voepel-Lewis T, Tait AR et al. Depth of sedation in children undergoing computed tomography: validity and reliability of the University of Michigan Sedation Scale (UMSS). Br J Anaesth 2002; 88: 241-245.

14 Malviya S, Voepel-Lewis T, Chiravuri SD et al. Does an objective system-based approach improve assessment of perioperative risk in children? A preliminary evaluation of the 'NARCO'. Br J Anaesth 2011; 106: $352-358$.

15 Cook NR. Statistical evaluation of prognostic vs diagnostic models: beyond the ROC curve. Clin Chem 2008; 54: 17-23.

16 Cohen J. A power primer. Psychol Bull 1992; 112: $155-159$.

17 Maund E, McDaid C, Rice S et al. Paracetamol and selective and non-selective non-steroidal anti-inflammatory drugs for the reduction in morphine-related side-effects after major surgery: a systematic review. $\mathrm{Br} \mathrm{J}$ Anaesth 2011; 106: 292-297.

18 Ong CK, Lirk P, Seymour RA et al. The efficacy of preemptive analgesia for acute postoperative pain management: a meta-analysis. Anesth Analg 2005; 100: 757-773.

19 Remy C, Marret E, Bonnet F. Effects of acetaminophen on morphine side-effects and consumption after major surgery: meta-analysis of randomized controlled trials. $\mathrm{Br} J$ Anaesth 2005; 94: 505-513.

20 De OliveiraGS, Jr, Agarwal D et al. Perioperative single dose ketorolac to prevent postoperative pain: a meta-analysis of randomized trials. Anesth Analg 2012; 114: 424 433.

21 Elia N, Lysakowski C, Tramer MR. Does multimodal analgesia with acetaminophen, nonsteroidal antiinflammatory drugs, or selective cyclooxygenase- 2 inhibitors and patient-controlled analgesia morphine offer advantages over morphine alone? Meta-analyses of randomized trials Anesthesiology 2005; 103: 1296-1304

22 Romsing J, Moiniche S, Mathiesen O et al. Reduction of opioid-related adverse events using opioid-sparing analgesia with COX-2 inhibitors lacks documentation: a systematic review. Acta Anaesthesiol Scand 2005; 49 133-142.

23 Marret E, Kurdi O, Zufferey P et al. Effects of nonsteroidal antiinflammatory drugs on patient-controlled analgesia morphine side effects: meta-analysis of randomized controlled trials. Anesthesiology 2005; 102 : 1249-1260.

24 Michelet D, Andreu-Gallien J, Bensalah T et al. A meta-analysis of the use of nonsteroidal antiinflammatory drugs for pediatric postoperative pain. Anesth Analg 2012; 114: 393-406.

25 American Society of Anesthesiologists Task Force on Acute Pain Management. Practice guidelines for acute pain management in the perioperative setting. Anesthesiology 2004; 100: 1573-1581

26 Smith HS, Lesar TS. Analgesic prescribing errors and associated medication characteristics. J Pain 2011; 12: 29-40.

27 Lynn LA, Curry JP. Patterns of unexpected in-hospital deaths: a root cause analysis. Patient Saf Surg 2011; 5: 3.

28 Fu ES, Downs JB, Schweiger JW et al. Supplemental oxygen impairs detection of hypoventilation by pulse oximetry. Chest 2004 ; 126: 1552-1558.

29 Overdyk FJ, Carter R, Maddox RR et al. Continuous oximetry/capnometry monitoring reveals frequent desaturation and bradypnea during patient-controlled analgesia. Anesth Analg 2007; 105 412-418.

30 Keidan I, Gravenstein D, Berkenstadt H et al. Supplemental oxygen compromises the use of pulse oximetry for detection of apnea and hypoventilation during sedation in simulated pediatric patients. Pediatrics 2008; 122: 293-298.

31 Sharek PJ, Parry G, Goldmann D et al. Performance characteristics of a methodology to quantify adverse events over time in hospitalized patients. Health Serv Res 2011; 46: 654 678.

32 Joint Commission. Safe use of opioids in hospitals. The Joint Commission Sentinel Event Alert, August 8, 2012;49. 